\title{
MELHORES PRÁTICAS DE ENFERMAGEM NA ATENÇÃO PRIMÁRIA À SAÚDE: RELATO DE AÇÃO PEDAGÓGICA
}

\author{
Glaucia Bohusch \\ Marina da Silva Sanes \\ Carine Vendrusculo \\ Fernanda Karla Metelski
}

\section{INTRODUÇÃO}

No contexto brasileiro, a Atenção Primária à Saúde (APS) é considerada a principal porta de entrada para o Sistema Único de Saúde (SUS), o qual deve ordenar a assistência/cuidado, além da formação em saúde. Para cumprir o seu papel dentro do SUS e estabelecer a comunicação entre todos os pontos da Rede de Atenção à Saúde (RAS), a APS - denominada de Atenção Básica (AB) no Brasil - desempenha algumas funções específicas, dentre elas: ser resolutiva e coordenar o cuidado. Tem como principal modelo de reorientação dos serviços a Estratégia Saúde da Família (eSF), ampliando o cuidado qualificado por meio de equipes de trabalho multiprofissionais, compostas por profissionais generalistas ${ }^{1}$.

$O$ trabalho da enfermagem ganha destaque na APS/AB pela atuação desse profissional, o qual agrega à técnica desenvolvida maior resolutividade ao dedicar parte considerável do tempo às ações cuidativo-educacionais, a exemplo da atenção à saúde da criança e pré-natal. O processo de trabalho do enfermeiro na APS ganha status protagonista, técnico-científica, legal e político, mediante práticas comprometidas com o bem-estar social, a partir das dimensões assistencial e gerencial. Sobre o cuidado de enfermagem, identifica-se como objeto de intervenção a pessoa, indivíduo e coletividades que tem necessidades relacionadas ao viver com saúde e/ou adoecimento, acionando um campo de conhecimentos da saúde coletiva e da prática assistencial dos enfermeiros, com destaque ao fortalecimento dos potenciais de saúde no ciclo vital e não apenas o foco na superação das doenças².

No Brasil, e especialmente no âmbito da Associação Brasileira de Enfermagem (ABEn), tem se ampliado o debate sobre as melhores práticas em enfermagem, como possibilidade de atender e fortalecer os princípios organizativos da RAS e consequentemente do SUS. Considera-se uma "melhor prática" aquela definida como técnica ou metodologia que, pela experiência ou investigação, possui confiabilidade comprovada para produzir um bom resultado. Consiste, portanto, no conhecimento sobre a prática que funciona em situações e contextos específicos, com a utilização racionada de recursos para atingir os resultados desejados, e que pode ser replicada em outras situações ou contextos ${ }^{3}$. 
Com base nessas reflexões, acredita-se que a enfermagem, como profissão que integra a equipe generalista da APS, precisa transcender a formação profissional atual, promovendo a rediscussão dos saberes essenciais de uma prática ampliada, pautada em evidências científicas. Em destaque também devem estar as ações que são específicas de cada profissão e que se complementam na busca da atenção integral e práticas colaborativas 4 .

Na perspectiva da formação dos profissionais de enfermagem no âmbito da graduação, as Diretrizes Curriculares Nacionais (DCN) para o Curso de Graduação em Enfermagem, homologadas em 07 de dezembro de 2001 pela Resolução ํo 03 de $7 / 11 / 2001^{5}$, orientam a formação do enfermeiro na direção de um perfil generalista, humano, crítico, reflexivo e orientado pelos princípios científicos ${ }^{6}$.

Assim, ao entender que o constructo de melhores práticas deve ser objeto de análise, reflexão e incorporação ao fazer clínico da enfermagem no contexto da APS, este manuscrito apresenta um relato de prática pedagógica sobre a temática "melhores práticas de enfermagem na APS", realizado em 2019, durante o $28^{\circ}$ Fórum Catarinense das Escolas de Enfermagem e promovido pela ABEn - Seção Santa Catarina.

\section{CAMINHO METODOLÓGICO}

A ABEn é uma organização civil não governamental, cuja criação data de agosto de 1926, sob a denominação de Associação Nacional de Enfermeiras Diplomadas (ANED). Após, em 1929, com o objetivo de ingressar no International Council of Nurses (ICN), recebeu a denominação de Associação Nacional de Enfermeiras Diplomadas Brasileiras (ANEDB). Assim permaneceu até 1944, quando passou a chamar-se Associação Brasileira de Enfermeiras Diplomadas (ABED), com a reforma do estatuto. Em 1954, a ABED recebeu a denominação de Associação Brasileira de Enfermagem ${ }^{7}$.

Desde então, a ABEn desempenha importante papel para a preservação da memória e desenvolvimento da cultura, relacionados à enfermagem brasileira, destacando-se pelas ações no contexto da educação em enfermagem, sendo uma das responsáveis pela criação do primeiro curso de graduação em enfermagem em SC, na UFSC, no ano de $1969{ }^{8}$.

A temática de melhores práticas em enfermagem esteve entre os tópicos de interesse e desenvolvimento por parte da gestão ABEn-SC (2016-2019), tanto que a Direção de Pesquisa, em parceria com a Universidade do Estado de Santa Catarina (UDESC) e outras instituições com cursos de enfermagem, desenvolveram a pesquisa: "Cuidado e Gestão em Enfermagem como Saberes na Rede de Atenção à Saúde: proposições para as boas práticas". Esse estudo teve, dentre seus objetivos, descrever as evidências utilizadas pelos enfermeiros nas práticas, no âmbito da eSF. 
Historicamente envolvida e participativa no tema educação em enfermagem, a ABEn- Seção Santa Catarina, realizou em junho de 2019, no município de Joaçaba, o 28ํㅜㄴ Fóm Catarinense das Escolas de Enfermagem, que teve com principal objetivo reunir estudantes, professores, coordenadores de curso e demais profissionais de enfermagem interessados em dialogar sobre as pautas pertinentes à formação em enfermagem. $O 28^{\circ}$ Fórum teve como temáticas: novas Diretrizes Curriculares Nacionais do Ensino Superior (DCNS), potencialidades e desafios do processo de ensino em enfermagem presencial e à distância e interprofissionalidade como eixo para a construção do Projeto Pedagógico Curricular. Parte integrante do evento, oficinas foram planejadas com a intencionalidade de abordar diferentes temáticas de modo teórico-prática e participativo.

A oficina de APS objetivou refletir sobre as práticas da enfermagem na APS, de modo a problematizar - de modo teórico-prático-vivencial - o fazer da enfermagem na APS, fornecendo ferramentas de apoio e qualificação à prática clínica de enfermeiros na APS.

A Oficina "Atenção Primária em Saúde: a enfermagem em foco" buscou refletir sobre a qualidade das práticas do enfermeiro na APS. Para tanto, foi estruturada em três momentos/questões disparadoras: (1) O que o grupo compreende sobre melhores práticas em enfermagem na APS?; (2) O que existe documentado sobre melhores práticas em enfermagem na APS?; (3)Como qualificar a prática clínica da enfermagem para que ela esteja filiada aos pressupostos das melhores práticas?

\section{RELATO DA EXPERIÊNCIA E REFLEXÕES}

\section{Momento 1: $O$ que o grupo compreende sobre melhores práticas em enfermagem na APS}

No primeiro momento da oficina foi realizada uma "dinâmica quebra-gelo" que teve por objetivo aproximar os participantes e facilitadoras, promovendo um clima de interação e descontração. Os participantes foram convidados a circular pelo espaço físico da sala de modo a responder a algumas perguntas. Foi apresentada uma primeira questão disparadora: "qual é o recurso que você mais utiliza para a construção da sua agenda pessoal ?" e os participantes se agruparam de acordo com a resposta escolhida. Este movimento estimulou o grupo a fazer escolhas e a conhecer as respostas dos demais participantes, percebendo o outro, identificando afinidades que aproximam e diferenças que evidenciam a riqueza de possibilidades existentes no conjunto. Outras perguntas foram lançadas, como "dentre os diferentes ciclos de vida, qual eu mais me identifico no momento de prestar o cuidado ?", dando continuidade ao movimento e estimulando comentários sobre as escolhas. Para todas as interrogações haviam opções de resposta. O último questionamento desta primeira 
dinâmica foi "em qual segmento você atuou por mais tempo na sua trajetória profissional?"

Esta pergunta permitiu que as facilitadoras da oficina fizessem uma discussão com os participantes da oficina sobre quais são os espaços que eles ocupam no cotidiano, e o quanto essas experiências contribuem para as suas compreensões e bagagens, concluindo e valorizando que cada um traz consigo o acúmulo de conhecimentos e vivências que reflete as próprias escolhas e decisões. Somos profissionais, mas também usuários, em alguns momentos podemos ser gestores, ou mesmo prestadores. Esses quatro segmentos constituintes do quadrilátero da formação, por vezes, não se reafirma como tal, ora faltando elementos para encaixar os vértices, ora com a intervenção de outros elementos em um mesmo segmento, configurando-se o que pode-se definir como um "prisma", para além de quadrilátero". As autoras ${ }^{9}$ indicam que as relações que se estabelecem no prisma, a partir dessas diferentes vivências e conhecimentos, contribuem para a construção dos sujeitos que compõem os diferentes segmentos. Elas são provocadas pela ação do quadrilátero, contudo, as relações dialógicas dos sujeitos geram oportunidades significativas de outros reflexos que mais se parecem com prismas, os quais traduzem melhor a complexidade, a riqueza e a potência dos movimentos de educação na saúde.

$\mathrm{Na}$ sequência, as facilitadoras buscaram conhecer quais eram as percepções e experiências dos participantes sobre o tema melhores práticas de enfermagem na APS, e para tanto, fizeram uso de uma metodologia ativa conhecida como "A Margarida". As metodologias ativas são metodologias de ensino-aprendizagem que permitem ao estudante ser o centro do processo pedagógico/andragógico e o professor o facilitador, orientador.

Os participantes foram convidados a se posicionar em torno de uma grande mesa com a intenção de refletir sobre sua prática profissional e estudantil. Foram utilizadas duas perguntas disparadoras para estimular o processo reflexivo dos participantes, sendo elas: (1) Você já utilizou alguma prática criativa na sua prática profissional? - em caso afirmativo - Como foi esta experiência? (2) Em quais elementos estão alicerçados o cuidado que você prescreve? Então eles receberam um círculo em papel cartolina, e foi solicitado ao grupo debater e escrever a partir do questionamento "o que vocês compreendem por "melhores práticas em enfermagem no contexto da APS"? Durante o debate cada participante pode expressar a sua opinião e falar sobre a sua compreensão a partir de uma palavra. Foram mencionadas: inclusão; vínculo; corresponsabilidade; resolutividade; trabalho em equipe; território; engajamento; prática colaborativa; empatia; acolhimento; adaptação.

O próximo passo foi o retorno para os seus lugares de assento na roda, quando receberam uma folha de papel em formato de pétala e foram convidados a escrever um exemplo de melhor prática, e as fontes utilizadas para a tomada de decisão. Entre os exemplos de melhores práticas foram citados: grupos (gestantes, hipertensos, diabéticos); consultas compartilhadas; visita domiciliar para idosos, e no puerpério, 
buscando a identificação de situações para resolver / melhorar / modificar; educação em saúde; atuar de modo conjunto com a escola; clínica ampliada; projeto Sérgio Arouca; palestra; e consulta de enfermagem. Entre as fontes utilizadas para a tomada de decisão foram elencadas: Política Nacional de Atenção Básica; Política Nacional de Humanização; Constituição Federal; Lei 8.080/90; documentos da Organização Mundial de Saúde e Organização Pan Americana de Saúde que tratam de assuntos como a interprofissionalidade; Programa Saúde na Escola; Recomendações; Políticas de Saúde (programas, estratégias); orientações do serviço; e, Plano Pedagógico do Curso.

À medida que os participantes iam apresentando, as facilitadoras iam recebendo as pétalas e procedendo à montagem da "Margarida" (figura 1). Até aquele momento não havia sido mencionado o motivo do formato da folha recebida. As facilitadoras expuseram para o grande grupo as experiências e percepções trazidas em cada pétala, preparando o grupo para o próximo momento da oficina que tratou sobre o tema das melhores práticas de enfermagem na APS. Os participantes foram convidados a refletir sobre suas práticas e compreensões à luz do referencial teórico a ser apresentado no segundo momento, observando se os seus exemplos e fontes de informação estão próximos ou distantes do que existe na literatura.

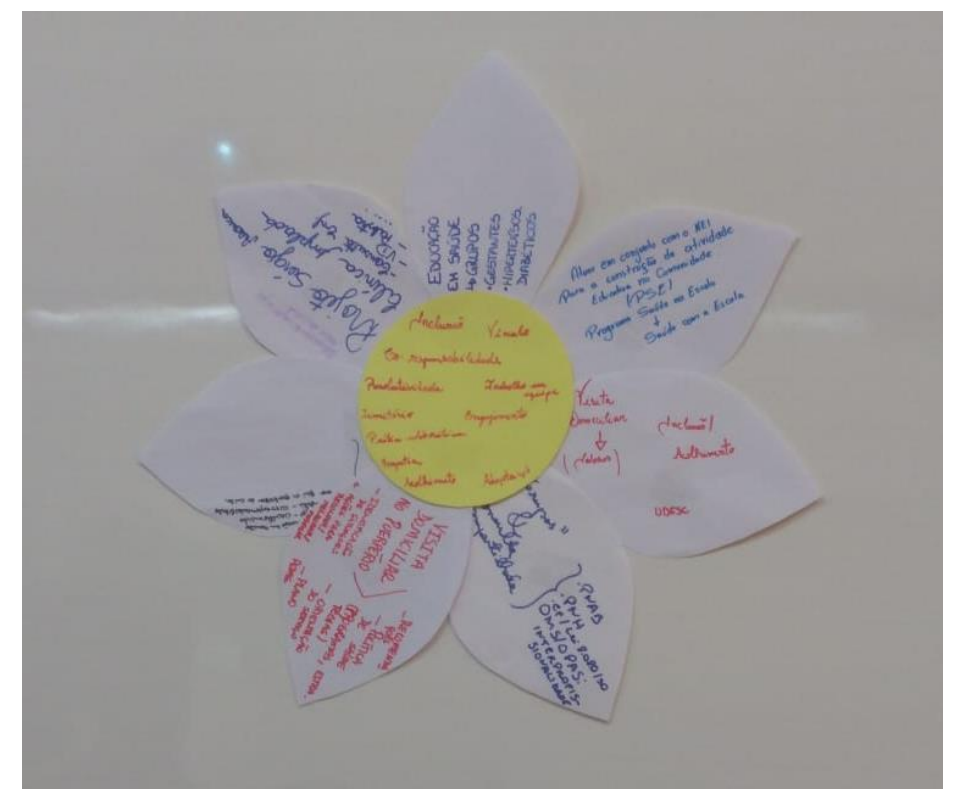

Figura 1 - A Margarida

Fonte: Participantes da Oficina, 2019.

Partiu-se do pressuposto de que a teoria emerge da prática, e, portanto, ambas devem ser aproximadas para que a aprendizagem tenha significado, e para que seja possível estabelecer relações entre o saber e o fazer profissional. Essa postura confere criticidade à atuação profissional, provocando reflexões instigadoras de mudanças e aguçando as inquietudes da prática profissional. Oportunizar aos 
participantes o compartilhamento de suas compreensões e experiências prévias confere sentido à construção coletiva que se propõe em uma oficina.

\section{Momento 2: 0 que existe documentado sobre melhores práticas em enfermagem na APS?}

Para essa construção coletiva, foi utilizada a exposição dialogada para interação com os participantes, buscando explorar conceitos e referenciais, bem como, comentando sobre resultados parciais da pesquisa sobre melhores práticas desenvolvida pela ABEn-SC.

A APS pode ser considerada o espaço onde os cuidados primordiais de saúde são desenvolvidos, e para tanto possui atributos essenciais (integralidade, acessibilidade, coordenação do cuidado e longitudinalidade), e atributos derivados (orientação familiar, competência cultural, e orientação comunitária), que juntos buscam a resolutividade da atenção. A APS situa-se no centro da RAS e, portanto, confere direcionalidade para as ações em saúde desenvolvidas ${ }^{1}$.

Nesse espaço, o trabalho do enfermeiro ganha destaque, ou seja, passando a ser um protagonista técnico-científico, legal e político, que desenvolve práticas comprometidas com o bem-estar social, considerando diferentes dimensões de sua prática profissional: assistencial, gerencial e educativa ${ }^{5}$. Ainda, tem a possibilidade de buscar formação a nível de stricto sensu para se tornar um Enfermeiro de Práticas Avançadas.

As melhores práticas são definidas pela Organização Mundial de Saúde ${ }^{3}$ como:

um conjunto de técnicas, processos e atividades, identificadas como as melhores para realizar determinada tarefa - por meio da experiência e da investigação, possuem confiabilidade comprovada para conduzir a um determinado resultado. Conhecimento sobre aquilo que funciona em situações e contextos específicos, sem a utilização desmesurada de recursos e que pode ser utilizado para desenvolver e implementar soluções adaptadas a problemas de saúde semelhantes noutras situações ou contextos.

Uma prática desenvolvida em um determinado contexto (serviço, comunidade ou na formação) que teve um resultado positivo e pode ser replicada em outros contextos e portanto, é considerada uma melhor prática. Melhor no sentido de que agregou, modificou, e como tal, não há a necessidade de ser uma prática considerada "padrão ouro" ${ }^{3}$, ainda que necessite sim estar apoiada em evidências, mas aquela que traga respostas. 
A prática baseada em evidências considera as melhores evidências acerca de determinada situação que se pretende melhorar, a expertise profissional, ou seja, os conhecimentos e experiência que cada profissional traz consigo sobre o que funciona bem, levando em conta os valores e preferências individuais, pois respeita os diferentes atores como corresponsáveis do seu processo de cuidado, e portanto, está centrada na pessoa ${ }^{3}$.

As práticas de cuidado dos enfermeiros na APS têm um significado importante, pois, neste campo, ocorre uma aproximação social e cultural com as necessidades da população, possibilitando a realização de práticas de cuidado mais coerentes e eficazes ${ }^{10}$. Com isso, os enfermeiros têm a oportunidade de adequar as suas ações na busca de uma saúde emancipadora e de direito universal ${ }^{11}$, mediante práticas que incentivem a construção de autonomia e do autocuidado, em que os usuários sejam protagonistas no seu projeto de cuidado. Deste modo, é possível contribuir com a busca de uma clínica ampliada ${ }^{11}$.

A qualificação clínica é uma questão sensível a enfermeiros que trabalham na APS. Muitas vezes, os profissionais não se sentem capacitados para executar atendimentos que exijam uma avaliação clínica devido à formação frágil neste quesito. Além disso, têm dificuldades para compreender sua atribuição na consulta de enfermagem fora das linhas de cuidado preconizadas pelo Ministério da Saúde ${ }^{12}$.

Estudo internacional indica que a ampliação da prática clínica dos enfermeiros na APS melhora o acesso e se configura em uma porta de entrada para o sistema de saúde $^{13}$.Sugere, ainda, bons resultados de saúde e satisfação dos usuários ${ }^{13}$.

Com isso, a necessidade de Educação Permanente nos serviços de saúde é fundamental para que os enfermeiros aprimorem a sua prática clínica na perspectiva da clínica ampliada, baseada em evidências científicas e experiências exitosas.

\section{Momento 3: Como qualificar a prática clínica da enfermagem para que ela esteja filiada aos pressupostos das melhores práticas?}

A qualificação da prática clínica requer o envolvimento dos sujeitos em movimentos contínuos, como educação permanente, capacitações, cursos de especialização, pesquisas em bases de dados, participação em grupos profissionais, seja de estudos ou associações, que promovem a disseminação e troca de informações. Uma prática de qualidade está alicerçada em fontes de informação fidedignas que apresentem as melhores evidências, experiência e expertise profissional, e o que tem significado e valor para cada pessoa ${ }^{3}$.

Assim, as facilitadoras iniciaram este momento apresentando ferramentas que exemplificam fontes de informação confiáveis e que podem ser utilizados no cotidiano do enfermeiro: Biblioteca Virtual em Saúde; Ministério da Saúde/Cadernos de Atenção Básica; Protocolos Clínicos e Diretrizes de Tratamento; National Institute for Health and Care Excellence; Plataforma Sabiá; Portal Baseado em Evidências; 
Telessaúde/SC; Livros; Sites de Instituições de Ensino como Universidade Federal de Santa Catarina, Universidade do Estado de Santa Catarina, Escola de Saúde Pública de Santa Catarina.

No mesmo sentido de colaborar com a aproximação com a temática de melhores práticas, a finalização da oficina objetivou proporcionar um momento de autorreflexão para os participantes, considerando um momento de pensar o quanto cada um reconhece na sua prática profissional as suas potencialidades, os seus desafios.

Realizando uma análise nesta perspectiva, é possível identificar meios de qualificação individual, coletiva e institucional. Isso é relevante na medida que os contextos das práticas clínicas da enfermagem na APS influenciam de modo direto ou indireto. Por exemplo, o quanto cada profissional é responsável pela sua própria formação? Estudar, ler, estar atento ao desenvolvimento científico da sua área, ter uma postura investigativa vinculada à prática profissional são ações de responsabilidade individual. Em outra via, qual a responsabilidade da gestão e da instituição na formação e qualificação profissional? Aqui, compreende-se como relevante a ideia de que os processos de formação e qualificação profissional necessitam ser incentivados e bem estruturados na política institucional. Isso pode ser legitimado pela Política Nacional de Educação Permanente e os seus desdobramentos nos serviços de saúde.

Assim, ao pensar melhores práticas da enfermagem na APS, é relevante refletir a partir de uma ideia situada sobre responsabilidades e ações. Para isso, as facilitadoras utilizaram uma matriz estratégica organizacional SWOT (strengths, weaknesses, opportunities, threats), muito utilizada no campo do Planejamento Estratégico-Situacional, que tem como objetivo de fazer análise de cenários internos e externos numa organização ${ }^{14}$. Na tradução para o português tem-se a matriz FOFA, cuja sigla deriva das palavras: fortalezas, oportunidades, fraquezas e ameaças.

Reservando-se todas as possibilidades que a matriz tem no campo do planejamento de organizações, o objetivo foi disparar a reflexão sobre o cada participante compreende como suas fortalezas, oportunidades, fraquezas e ameaças, considerando um contexto interno (individual) e externo (institucional), no seu processo laboral.

A matriz FOFA (figura 2) trata-se de um instrumento utilizado para planejamento que possibilita a visualização organizada de pontos fortes e fracos, favorecendo a avaliação do contexto, da estrutura e do desempenho, possibilitando conhecer características e particularidades. Os pontos fortes são as fortalezas próprias e as oportunidades externas, enquanto que os pontos fracos são as fraquezas próprias e as ameaças externas ${ }^{15}$. 


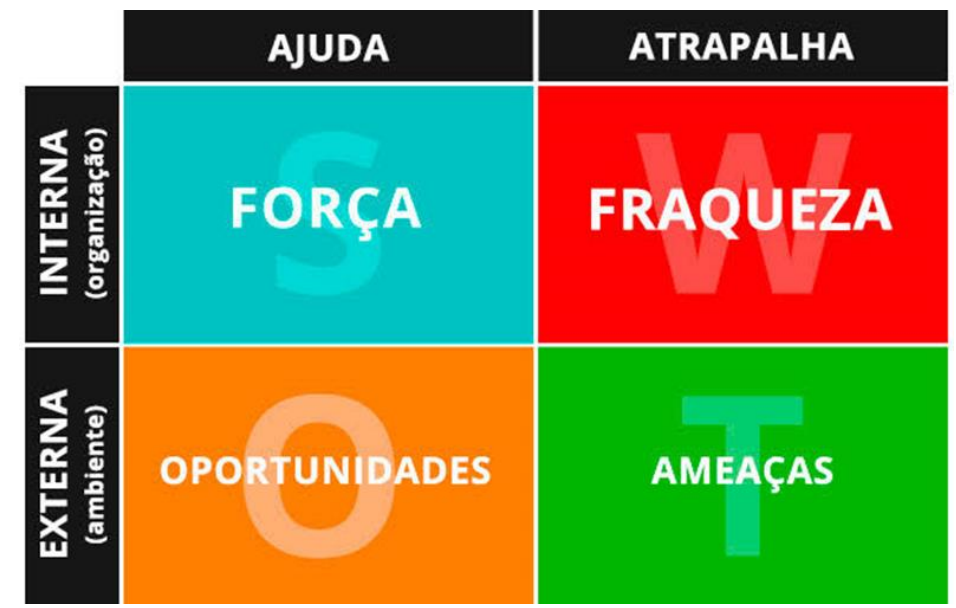

Figura 2 - Matriz FOFA

Fonte: https://www.ignicaodigital.com.br/como-usar-analise-swot-para-avaliar-minhaequipe/

Com o auxílio da matriz FOFA, as facilitadoras disponibilizaram uma cópia da matriz para cada participante, de modo que cada um pudesse escrever a sua matriz. Em seguida, conduziu-se um momento reflexivo sobre como os elementos internos (forças e ameaças) e os elementos externos (oportunidades e ameaças) contribuem e/ou atrapalham a qualificação profissional, especialmente melhores práticas.

Nesse sentido, ao problematizar sobre a matriz que cada um consegue visualizar sobre a sua prática, ela também consegue demonstrar se estes elementos são mais sensíveis ou não às ações individuais, coletivas e/ou institucionais. Com o uso da matriz, é possível identificar como determinada força pode ser impulsionada ou como determinada fraqueza possa ser neutralizada. Ao realizar este exercício na oficina, cada participante consegue visualizar na sua matriz se as forças, oportunidades, fraquezas e ameaças e se são elementos mais vinculados às próprias responsabilidades ou se exigem ações coletivas, da equipe ou até da instituição.

Encerrando este momento, os participantes assistiram um vídeo com imagens da Aurora Boreal ( ) refletindo que assim como acontece com o planeta Terra, 0 quanto as ameaças externas podem ser oportunidades quando estamos preparados mental e emocionalmente para lidar com situações desafiadoras.

\section{CONSIDERAÇÕES FINAIS}

A ABEn-SC busca aproximar suas ações das competências gerais e específicas descritas nas DCN, de modo a cumprir o seu papel político cultural junto aos profissionais de enfermagem do Brasil, colaborando com o desenvolvimento de uma enfermagem de qualidade, respaldada legal e cientificamente, e que contribua para a melhoria das condições de vida e saúde da população. Nessa direção, a Oficina 
realizada durante o Fórum das Escolas em 2019, contribuiu para a reflexão e, possivelmente, terá algum impacto na ação dos estudantes e profissionais envolvidos.

Entende-se que o desafio principal reside na necessidade de sensibilização dos enfermeiros quanto à importância da qualificação da sua prática na APS, com vistas ao desenvolvimento de um cuidado pautado em evidências, resolutivo e de qualidade. Esses aspectos também contribuirão com a resolutividade da APS, demonstrando compromisso social da profissão com necessidades dos indivíduos, famílias e coletividades.

Longe de ser um cenário de cuidado comum a todas as profissões, a atuação na APS exige cada vez mais preparo e formação específica devido à complexidade do contexto e o caráter ordenador da RAS. Assim, o conhecimento técnico-científico, legal e humano; o domínio de instrumentos de cuidado, informação e gestão; o desenvolvimento de competências profissionais; a participação em movimentos de educação permanente; a busca por cursos de residência, especialização e mestrado; e a participação em eventos que oportunizem a trocas de saberes e experiências constituem requisitos primordiais para o desenvolvimento de um cuidado de qualidade, configurando-se como fonte técnico-científica para o desenvolvimento de melhores práticas.

\section{REFERÊNCIAS}

Ministério da Saúde (BR). Secretaria. Política Nacional de Atenção Básica. Brasília (DF); 2017 [internet][acesso em 03 Mai 2019]. Disponível em: http://www.foa.unesp.br/home/pos/ppgops/portaria-n-2436.pdf.

Backes DS, Erdmann AL, Büscher A. O papel profissional do enfermeiro no Sistema Único de Saúde: da saúde comunitária à estratégia de saúde da família. Ciência \& Saúde Coletiva. 2012; 17(1):223-230. doi: http://dx.doi.org/10.1590/S141381232012000100024.

Organização Mundial de Saúde (OMS). Guia para a Documentação e Partilha das Melhores Práticas em Programas de Saúde.OMS - Escritório Regional Africano Brazzaville; 2008. [internet][acesso em 03 JUN 2019]. Disponível em: http://afrolib.afro.who.int/documents/2009/pt/GuiaMelhoresPratica.pdf.

World Health Organization (WHO). Framework for action on interprofessional education and collaborative practice. Geneva: WHO, 2010. Disponível em: https://www.who.int/hrh/resources/framework_action/en/

Brasil. Resolução CNE/CES n-1133 de 2001. Diretrizes Curriculares Nacionais dos Cursos de Graduação em Enfermagem, Medicina e Nutrição. Diário Oficial da União, Brasília, 03 de 10 de 2001. Seção 1E, p. 131. Disponível em: 
http://portal.mec.gov.br/dmdocuments/ces1133.pdf.

Santos, SSC. Currículos de enfermagem do Brasil e as diretrizes - novas perspectivas. Rev Bras Enferm. 2003;56(4):361-64. doi: http://dx.doi.org/10.1590/S003471672003000400009 .

Santos TCF. A ABEn e a preservação da memória profissional: implantação do Centro de Memória da Enfermagem Brasileira. Rev Bras Enferm [Internet] 2013; 66(esp):16570. doi: http://dx.doi.org/10.1590/S0034-71672013000700021.

Vendruscolo C, Mafissoni AL, Pértille F, et al. Ensino Superior e Associação Brasileira de Enfermagem: contribuições para o desenvolvimento e as memórias da profissão no Oeste de Santa Catarina. Here; 2018;9(2):122-30.

Vendruscolo, C; Do Prado, ML; Kleba, ME. Reorientação do ensino no sus: para além do quadrilátero, o prisma da educação. Reflexão e Ação. 2016; 24(3): 246-260. doi: http://dx.doi.org/10.17058/rea.v24i3.5420.

Acioli, S. et al. Práticas de cuidado: o papel do enfermeiro na atenção básica. Revista Enfermagem UERJ. 2014; 22(5): 637-42. doi: http://dx.doi.org/10.12957/reuerj.2014.12338.

Campos, CMS. et al. Práticas emancipatórias de enfermeiros na atenção básica à saúde: a visita domiciliar como instrumento de reconhecimento de necessidades de saúde. Revista da Escola de Enfermagem da USP. 2014; 48(spe): 119-25. doi: http://dx.doi.org/10.1590/S0080-623420140000600017.

Bohusch, G. Práticas dos enfermeiros no atendimento à demanda espontânea em equipes de saúde da família no município do Rio de Janeiro. Rio de Janeiro. Dissertação [ Mestrado]- Universidade do Estado do Rio de Janeiro;2019.

Laurant, M. et al. Nurses as substitutes for doctors in primary care. Cochrane Library. 2018. Doi: https://doi.org/10.1002/14651858.CD001271.pub3

Souza e Souza L, et al. Matriz SWOT como ferramenta de gestão para melhoria da assistência de enfermagem: estudo de caso em um hospital de ensino. Rev Elet GS. 2013 4(1):1633-4. Available from: http://periodicos.unb.br/index.php/rgs/article/view/207

Gomide M. Fortalezas, Oportunidades, Fraquezas e Ameaças (Matriz FOFA) de uma Comunidade Ribeirinha Sul-Amazônica na perspectiva da Análise de Redes Sociais: aportes para a Atenção Básica à Saúde. Cad. Saúde Colet., 2015; 23(3):222-230. doi: http://dx.doi.org/10.1590/1414-462X201500030089. 